\title{
A Novel Tactile Sensor for Detecting Lumps in Breast Tissue
}

\author{
Mehmet Ayyildiz ${ }^{1}$, Burak Guclu ${ }^{2}$, Mustafa Z. Yildiz ${ }^{2}$, and Cagatay Basdogan ${ }^{1}$ \\ ${ }^{1}$ College of Engineering, Koc University, Istanbul, 34450 Turkey \\ ${ }^{2}$ Biomedical Engineering Institute, Boğaziçi University, Istanbul, 34684 Turkey \\ mayyildiz@ku.edu.tr, burak.guclu@boun.edu.tr, \\ mustafa.yildiz@boun.edu.tr, cbasdogan@ku.edu.tr
}

\begin{abstract}
We developed a compact tactile sensor in order to guide the clinician or the self-user for non-invasive detection of lumps. The new design has an advantage over the existing discrete tactile sensors and detection methods by efficiently sensing force distribution over an area without any side effects. The sensor consists of $10 \times 10$ infrared emitter-detector pairs, a silicon-rubber elastic pad, and a contoured tactile interface ( $25 \times 21$ moving pins) for palpating threedimensional objects. To demonstrate the practical use of the sensor, first a cylindrical tissue-like silicon phantom was prepared, then a $13 \mathrm{~mm}$ diameter rigid spherical object was placed at varying depths of 0-20 $\mathrm{mm}$ to simulate cancerous lumps in breast tissue, and finally the tactile sensor was systematically pressed on the phantom to successfully detect the lumps for compression depths of 10$24 \mathrm{~mm}$. The location and the estimated radius of each lump were calculated from the recorded tactile images.
\end{abstract}

Keywords: Optical array sensor, lump detection, breast cancer, tactile mapping, artificial palpation, haptics.

\section{Introduction}

Cancer, a foremost cause of death worldwide, is accounted for 7.4 million deaths (around 13\% of all deaths) in 2004. [1] In Europe, 3,191,600 cancer diagnoses (excluding nonmelanoma skin cancers) and 1,703,000 deaths in consequence of cancer were recorded in 2006. Breast cancer was the most widespread type of all the cancer cases (a total of 429,900 cases account for 13.5\%). [2] Since the incidence rate of breast cancer is significantly high, early detection becomes a vital issue. However, the majority of the people in developing countries have insufficient access to screening and diagnostic medical equipment for the detection. [3] Although various sensing methods and devices have been developed, only a few has been accepted for standard clinical use. Tactile imaging is one of the potential methods to address the needs in breast cancer screening and diagnostics cost-effectively. [4] Mammography, one of the most popular diagnostic techniques in breast examination is unable to examine breast tissue near the chest wall and axilla. Palpation complements mammography by finding lumps which are the most common symptoms of breast cancer [5]. It was also found that as many as $12-15 \%$ of breast cancers that were detected by physical 
examination were not apparent on mammograms. [5-7] In conventional palpation, hands are used to evaluate the location, size, shape, and stiffness of breast tissue. However, it is not possible to obtain quantitative, objective and firm information in traditional hand palpation. [8-10] There are more quantitative methods, but they have some disadvantages. In X-ray computed tomography, excessive exposure to radiation creates an additional risk of cancer. [11] Likewise, magnetic resonance imaging (MRI) generates a powerful magnetic field and hence orthopedic implants, materials or devices in the body of patient or in the environment may cause hazardous situations. [12] In this study, a tactile sensor was developed for the quantitative assessment of lumps in soft tissue noninvasively. The proposed approach has no side effects and unlike in other diagnostic techniques mentioned above, the tactile sensor can be used at home for self-examination. Additionally, during the neoadjuvant chemotheraphy, the current design can provide regular information about the response of the patient to the treatment. Here, we present the design details of our system and the initial results of the lump detection experiments performed with a tissue-like silicon phantom.

\section{Materials and Methods}

\subsection{Tactile Sensor and Processor Module}

The tactile sensor consisted of infrared emitter-detector pairs (QRD1313, Reflective Object Sensor; Fairchild Semiconductor) arranged in a $10 \times 10$ array. The entire sensor was housed in an aluminum casing with a square base (Fig. 1). The side length of the square base is $9.2 \mathrm{~cm}$, and the height of sensor is $3.0 \mathrm{~cm}$. The sensor elements were covered by silicon-rubber tactile pad with outer surface dyed in black to block ambient light. The IR light rays emitted from the LEDs reflect off from the white inner surface of the rubber pad and their intensity is measured by the detectors. This reflection is modulated by force applied to the pad's outer surface, which is deflected towards the sensor element with the mechanical effect of the force. The force was transmitted from the palpation object to the pad via rigid pins which conformed to the contour of the object. The processing module received light-intensity data from each sensor element and transmitted this data array via a single analog channel by using
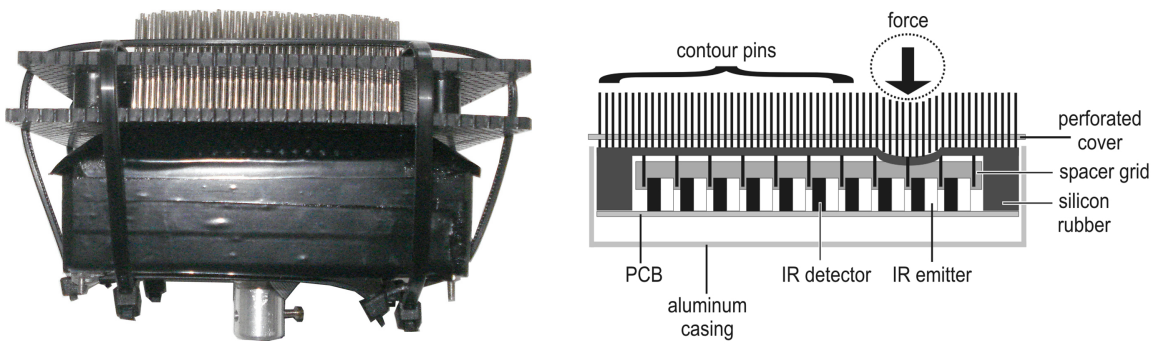

Fig. 1. a. Our tactile sensor. b. Cross section of the tactile sensor. 
time-division multiplexing (TDM). The output from the entire array took approximately $10.9 \mathrm{~ms}$; and therefore, the theoretical limit for the scan rate is $91.7 \mathrm{~Hz}$ in the presented design. The beginning of each packet cycle was signaled by a synchronization pulse. The processing module also amplified the multiplexed data and applied offset shifting to match the input range of the data-acquisition system.

\subsection{Data Acquisition and Calibration}

The sensor data was acquired by a 16-bit ADC card (NI6034; National Instruments) connected to a PC. The sampling rate was $100 \mathrm{kHz}$. This yielded 10-11 data points for each sensor element in every multiplexing cycle. Each sensor element was calibrated in the range of $0-5 \mathrm{~N}$ forces. The calibration was performed by a micromanipulator (WPI) and a digital balance (Kern). A software code in C language was developed in Visual C++ 6.0, (Microsoft) for sensor calibration, offset nulling, reading sensor array data, spatial interpolation, and outputting interpolated data. The sensor data was spatially interpolated to give an output array of 100 by 100 elements. The spatial interpolation was performed successively along the $\mathrm{x}$ - and $\mathrm{y}$-dimensions by up-sampling and then low-pass filtering to remove undesired spectral images. The low-pass filter was a 29th-order digital FIR filter designed with Kaiser Window (beta: 5), and it could achieve $65-\mathrm{dB}$ attenuation in the stop band. The spatial cutoff frequency was 5 cycles per linear length of the tactile membrane (i.e. $0.82 \mathrm{cycles} / \mathrm{cm}$ ). The scan rate achieved by the compiled program was $33 \mathrm{~Hz}$.

\subsection{Phantom Experiments and Analyses}

Lump detection experiments were conducted by a compression device consists of a moving shuttle on a power screw driven by a step motor (Fig. 2). Tactile array sensor was attached to the moving shuttle, which compressed a tissue-like cylindrical silicone phantom to varying depths of 10, 15, 20, and $24 \mathrm{~mm}$. The Young's modulus of the silicon sample was measured as $44 \mathrm{kPa}$ by compressing the sample slowly (rate: $0.5 \mathrm{~mm} / \mathrm{s}$ ) up to $20 \%$ strain. A rigid marble sphere having a radius of $6.5 \mathrm{~mm}$ was embedded into the silicon phantom (Fig. 3) at varying depths of 0, 4, 8, 12, 16, $20 \mathrm{~mm}$ to simulate a cancerous lump in a soft tissue. For each depth, the compression experiment was repeated 10 times. Additionally, 10 control measurements were performed at each compression depth without the simulated lump. The statistical differences between the interpolated sensor data and the control data were tested by Bonferroni-corrected two-sample t-test. To plot the sensor images, only the force values at pixels which were significantly different than that of the control experiments were selected and used in color coding (Fig. 4). Lump parameters were calculated based on the image data. Specifically, the peak force, and its pixel coordinates, the coordinates of the force centroid of the lump, and the estimated lump radius were all determined with respect to the control data. The lump radius was defined as the average distance between the pixel coordinates of the force centroid and the pixel coordinates which had force values greater than one tenth of the peak force. 


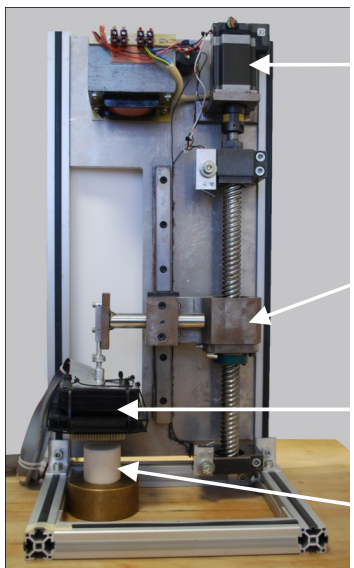

Fig. 2. The experimental set-up

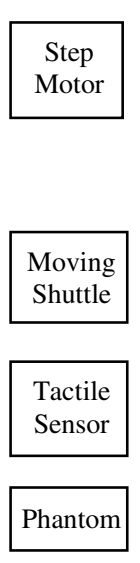

Fig. 3. The silicon phantom and definition of depth (D) in our experiments

\section{Results}

Fig. 5 shows the peak force read by the tactile array versus the depth of the lump with the compression depth of the tactile sensor used as an additional control parameter. As the depth of the simulated lump increases, the peak force decreases. However, peak forces at $20 \mathrm{~mm}$ lump depth are greater than those at $16 \mathrm{~mm}$, because the lumps at $20 \mathrm{~mm}$ are closer to the bottom surface, which acts as a boundary and increases the magnitude of the peak forces. The same figure also suggests that better detection of the lump is achieved at higher compression depths. The $\mathrm{x}$ and the $\mathrm{y}$ coordinates of the peak force detected by the tactile sensor with respect to the control data and the force centroid estimated from the distributed differential force response are plotted for the

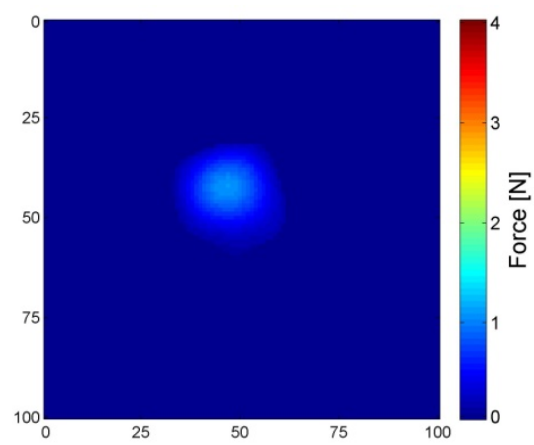

Fig. 4. An image of interpolated sensor data. Fig. 5. The peak force detected by the tactile The simulated lump was at $4 \mathrm{~mm}$ depth and the sensor versus the lump depth for the different compression depth was $15 \mathrm{~mm}$.

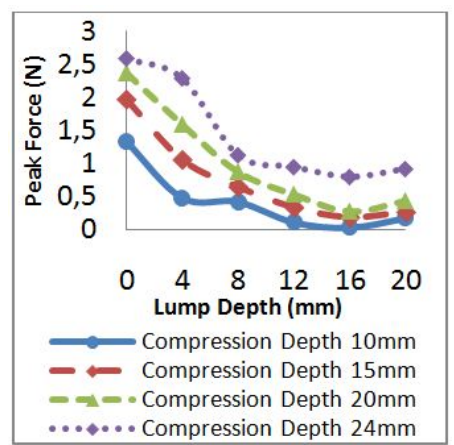

compression depths of the silicon phantom 

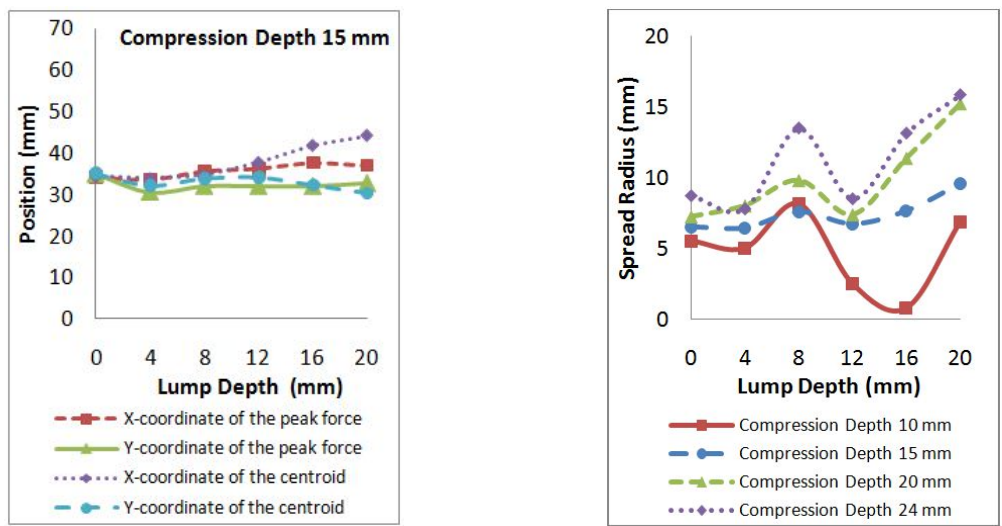

Fig. 6. The position of the peak force and the Fig. 7. The spread radius of the simulated force centroid for the different lump depths lump versus the lump depth

different lump depths in Fig. 6. As shown in the figure, the results are highly consistent. Furthermore, Fig. 7 shows that the spread radius of the simulated lump increases as the silicon phantom is compressed more, exciding the ideal value of $6.5 \mathrm{~mm}$ (radius of the spherical marble placed into the silicon) for the lump depths greater than $12 \mathrm{~mm}$. This is again due to the influence of the ground reaction forces caused by the lower boundary (bottom surface) of the silicon phantom.

\section{Conclusion and Future Work}

The aim of this study was to develop a prototype tactile array sensor which can be used by clinicians and home user to detect lumps in soft breast tissue. To evaluate the hardware, software and computational performance of the sensor, some preliminary compression experiments were performed with a tissue-like silicon phantom and a small spherical rigid object, imitating a cancerous lump, placed inside the phantom at various depths. The tactile sensor successfully detected all the lumps placed at the different depths, but for deeper lumps, more compression of the silicon phantom was required. However, as the compression depth was increased, the boundary conditions affected the force response of the tactile sensor. Based on the experimental results, we determined the optimum compression depth as $15 \mathrm{~mm}$ for the accurate detection of the lumps placed at varying depths (0 to $20 \mathrm{~mm})$ in this study. In the future, we plan to perform more experiments to characterize the static and dynamic performance of the tactile array and test the device on patients with breast cancer and compare the results with those acquired by conventional methods.

\section{References}

1. Organization, W. H. World Health Statistics 2009. World Health Organization, Geneva (2009)

2. Ferlay, J., Autier, P., Boniol, M., Heanue, M., Colombet, M., Boyle, P.: Estimates of the cancer incidence and mortality in Europe in 2006. Ann Oncol. 18(3), 581-592 (2007) 
3. Sarvazyan, A., Egorov, V.: Cost-Effective Screening for Breast Cancer Worldwide: Current State and Future Directions. Breast Cancer: Basic and Clinical Research, 91-99 (2008)

4. Lang, P.: Optical Tactile Sensor for Medical Palpation. In: The Thirty-Fourth London District Science and Technology Conference, pp. 1-5 (March 2004)

5. Wang, Y., Nguyen, C., Srikanchana, R., Geng, Z., Freedman, M.T.: Tactile Mapping of Palpable Abnormalities for Breast Cancer Diagnosis. In: Proc. of the Int. Conf. on Robotics and Automation, pp. 1305-1309 (May 1999)

6. Zeng, J., Wang, Y., Freedman, M.T., Mun, S.K.: Finger Tracking for Breast Palpation Quantification using Color Image Features. SPIE J. of Opt. Eng. 36(12), 3455-3461 (1997)

7. Haagensen, C.D.: Diseases of the Breast, 3rd edn. Saunders, Philadelphia (1986)

8. Kitagawa, M., Okamura, A.M., Bertha, B.T., Gott, V.L., Baumgartner, W.A.: Analysis of Suture Manipulation Forces for Teleoperation with Force Feedback. In: Dohi, T., Kikinis, R. (eds.) MICCAI 2002. LNCS, vol. 2488, pp. 155-162. Springer, Heidelberg (2002)

9. Ohtsuka, T., Furuse, A., Kohno, T., Nakajima, J., Yagyu, K., Omata, S.: New Tactile Sensor Techniques for Localization of Pulmonary Nodules. International Surgery 82, 12-14 (1997)

10. Wellman, P.S., Dalton, E.P., Krag, D., Kern, K.A., Howe, R.D.: Tactile Imaging of Breast Masses: First Clinical Report. Archives of Surgery 136, 204-208 (2001)

11. Carmichael, A., Sami, A., Dixon, J.: Breast cancer risk among the survivors of atomic bomb and patients exposed to therapeutic ionising radiation. European Journal of Surgical Oncology 29(5), 475-479

12. Shellock, F.G.: Biomedical Implants and Devices: Assessment of Magnetic Field Interactions with a 3.0-Tesla MR System. Journal Of Magnetic Resonance Imaging (2002) 